\title{
Calibration of the pulsed electroacoustic technique in the presence of trapped charge
}

\author{
G Chen ${ }^{1}$, Y L Chong ${ }^{2}$ and $M F^{3}$ \\ ${ }^{1}$ School of Electronics and Computer Science, University of Southampton, UK \\ 2 Data Storage Institute, Singapore \\ ${ }^{3}$ Department of Engineering, University of Leicester, UK
}

Received 6 January 2006, in final form 7 May 2006

Published 21 June 2006

Online at stacks.iop.org/MST/17/1974

\begin{abstract}
The influence of pulse voltage on the accuracy of charge density distribution in the pulsed electroacoustic technique (PEA) is discussed. It is shown that significant error can be introduced if a low dc voltage and high pulse voltage are used to calibrate charge density. However, our main focus in the present paper is to deal with one of the practical situations where space charge exists in the material prior to any measurements. The conventional calibration method can no longer be used to calibrate the charge density due to the interference by the charge on the electrode induced by space charge. A method has been proposed which is based on two measurements. Firstly, the sample containing the charge is measured without any applied voltage. The second measurement is carried out with a small external applied voltage. The applied voltage should be small enough so there is no disturbance of the existing charge in the sample. The difference of the two measurements can be used for calibration. An additional advantage of the proposed method avoids the influence of the pulse voltage on calibration and therefore gives a more accurate representation of space charge. The proposed method has been validated.
\end{abstract}

Keywords: space charge measurement, pulsed electroacoustic technique, charge calibration, pulse voltage, trapped charge, solid dielectrics

(Some figures in this article are in colour only in the electronic version)

\section{Introduction}

Solid polymeric materials, such as polyethylene (PE), crosslinked polyethylene (XLPE) and polypropylene (PP), are now being widely used in high-voltage power apparatus as insulators. The advantages of such materials are their high dielectric strength and electrical resistivity combined with good physical properties, such as resistance to cracking and moisture penetration. However, under certain operating conditions, their good electrical insulation properties may become degraded. For example, trapped or lowmobility electrically charged species within the bulk can give rise to space charge, resulting in localized electric stress enhancement. This can cause further concentration of charge and lead to premature failure of the material $[1,2]$

Recent developments in space charge measurement techniques have enabled the space charge in solid materials to be determined non-destructively. Three main techniques have been adopted worldwide by cable manufacturers and research institutes. They are the pulsed electroacoustic (PEA) method [3, 4], the laser-induced pressure pulse (LIPP) method [5, 6] and the thermal step or pulse method [7, 8]. Although the detailed principles for each method are different, which can be found in the literature, one thing that is common to the above methods is that they all need calibration before the quantitative space charge density can be obtained. A lot of papers have been published 

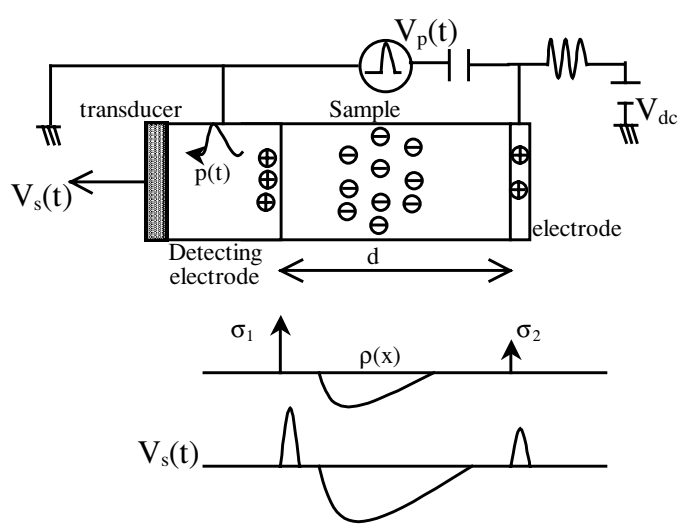

Figure 1. Schematic diagram of the PEA system.

over the years on space charge in polymeric insulations [9-19].

The PEA technique is more common than the other two techniques due to its simplicity in structure, low cost and its ease of implementation. In the present paper a normal procedure used for charge density calibration using the PEA technique is briefly described; the effect of the pulse voltage on quantitative charge distribution is discussed. Our main focus is to deal with one of the practical situations where space charge exists in the material prior to any measurements. After discussing the possible error that may occur with the existing method, a method based on two-step measurements is proposed. An experiment is devised to validate the proposed method and the result shows that the method is accurate and reliable.

\section{PEA system model and its frequency representation}

A schematic diagram of a PEA system is shown in figure 1 . In summary, acoustic waves are produced at charge layers at both electrodes and internal charge when an electrical pulse is applied to a sample. The acoustic signals are detected by a piezo-electric transducer. The electric signal obtained in the time domain represents the charge distribution [3]:

$$
V_{s}(t)=K\left[\sigma_{1}+\sigma_{2}+v_{\mathrm{sa}} \Delta T \rho\left(x=v_{\mathrm{sa}} t\right)\right] e_{\mathrm{p}},
$$

where $\sigma_{1}$ and $\sigma_{2}$ are the surface charges at the electrodes, $v_{\mathrm{sa}}$ is the sound velocity through the material, $\Delta T$ is the width of the pulse, $\rho$ is the bulk charge and $e_{\mathrm{p}}$ is the amplitude of the pulse voltage.

The quantitative charge analysis needs the system to be calibrated, i.e. $K$ needs to be determined. $K$ is determined by many factors such as acoustic properties of materials encountered by acoustic waves, and electrical parameters of the measuring system. In theory, it is extremely difficult to obtain an analytical solution due to the complexity of the system. In practice, it is often determined experimentally. For a planar sample if there is no bulk charge in the material the third term in equation (1) is zero; therefore, the output signal at the electrodes can be written as

$$
\begin{aligned}
& V_{1}=K \sigma_{1} e_{\mathrm{p}}, \\
& V_{2}=K \sigma_{2} e_{\mathrm{p}} .
\end{aligned}
$$

Both equations can be used to determine the constant $K$ if the acoustic attenuation and dispersion are negligible. However, in practice (equation (2)), the signal from the electrode closer to the transducer is often used as the acoustic signal experiences less loss.

A PEA system has been implicitly viewed as a linear time invariant (LTI) [20] where deconvolution was applied. Recently, a more detailed model has been proposed [21]. As this is important from a calibration point of view, it is worth reviewing the model briefly. It is well known [22] that the impulse response $h(t)$ of the LTI system is simply the output when the input is the unit impulse sequence $\delta(t)$ and the impulse response is a complete characterization of any LTI system. Convolution is the general formula that allows us to compute the output $y(t)$ from the input $x(t)$ for any LTI system.

In mathematical terms it can be represented by

$$
y(t)=\int x(\tau) h(t-\tau) \mathrm{d} \tau=x(t)^{*} h(t),
$$

where * represents an operation of the convolution.

In practice, the signal detected by the sensor is small and needs to be amplified. The sensor and the input impedance of the amplifier constitute a typical high-pass filter; this filter is another LTI system. Therefore the PEA system including the detecting circuit can be viewed as two LTI systems connected in cascade. One of the important properties of LTI systems is that the cascaded LTI systems behave as a new LTI system [22]. In a cascaded connection of two LTI systems, the output of the first system is the input of the second system, and the overall output of the cascaded system is the output of the second system. Assuming that the impulse response of the first LTI system is $h_{1}(t)$ and the second LTI system is $h_{2}(t)$, the overall impulse response of the cascaded system will be $h_{1}(t) * h_{2}(t)$. Therefore equation (4) is still valid even including the response of the detecting circuit.

It has been found [23] that an easy computation can be achieved by converting the signal from time domain to frequency domain. Equation (4) in the frequency domain can be represented as

$$
Y(f)=X(f) H(f),
$$

where $H(f)$ is the impulse response of the system, including the sensor and amplifier in the frequency domain.

In practice it is impossible to produce a unit impulse $\delta(t)$ which means $H(f)$ cannot be found. However, from equation (5), the characteristics of the system can be obtained by injecting a known $X(f)$ and measuring $Y(f)$. In order to obtain a broad spectrum, a narrow electric pulse is often applied to the system. Once $H(f)$ is obtained, its effect on the output signal can be removed (known as deconvolution).

\section{Calibration}

Calibration is important if quantitative charge distributions are required. This becomes more important as the understanding of the effect of the space charge on electrical ageing improves. There are several methods that can be used depending on the actual sample in question. In some cases it is not an easy task to perform a calibration. In the following sections calibration procedures have been considered according to the sample status. 


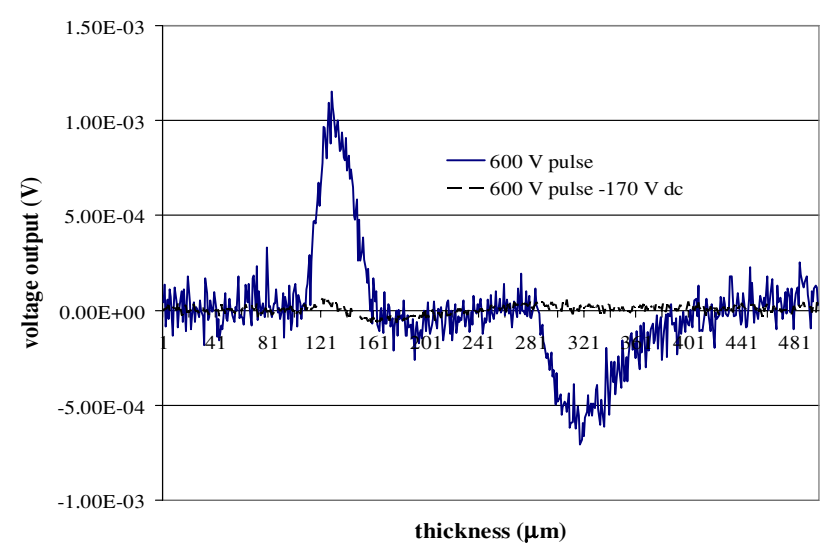

Figure 2. Effect of pulse voltage.

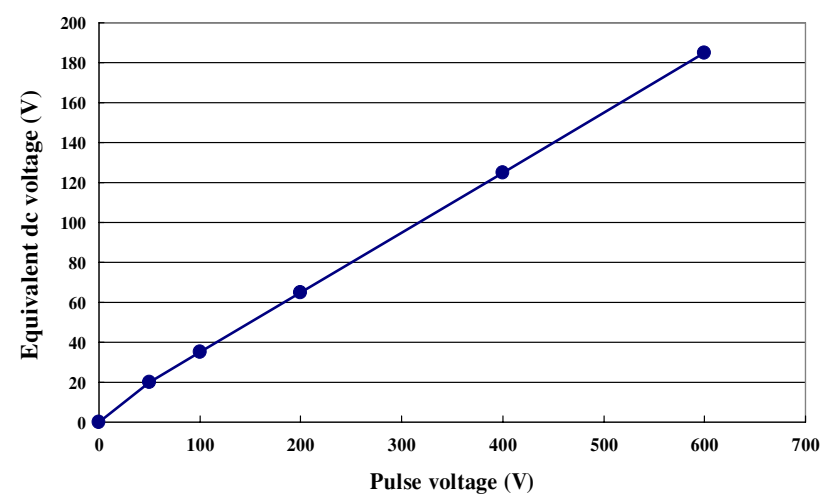

Figure 3. Pulse voltage and its equivalent dc voltage.

\subsection{Sample without space charge}

This is the situation for most of the measurements. If the PEA system is well constructed the noise level can be minimized. Based on equation (5), the narrow pulse itself can be used to calibrate the signal. Here the surface charge $\sigma_{1}$ is produced by the applied pulse voltage. The actual pulse voltage on the sample is related to the capacitance ratio of decoupling capacitor and sample. This means that although $\sigma_{1}$ is proportional to the pulse voltage the exact value cannot be easily obtained due to the influence of stray capacitance. Additionally, $\sigma_{1}$ will change with sample (material, electrode size, thickness etc). To solve this problem, an experiment has been devised. Applying a dc voltage with opposite polarity to the pulse voltage, the induced charges on the electrodes cancel each other. By adjusting the dc voltage, it is possible to cancel the effect of the pulse voltage. Figure 2 shows an output signal from a $600 \mathrm{~V}$ pulse. By applying a dc voltage of $170 \mathrm{~V}$ in the opposite direction, the peak of the output signal is almost cancelled. The pulse generator has a width of 5 ns with a voltage range from 50 to $600 \mathrm{~V}$.

Performing a series of such tests with varying pulse voltage amplitudes, the corresponding dc voltage can be found as shown in figure 3 . As expected, for a particular sample a linear relationship is obtained.

The above relationship between the pulse voltage and its equivalent dc voltage can be used to improve the accuracy of the charge analysis. For example, if a $600 \mathrm{~V}$ pulse voltage

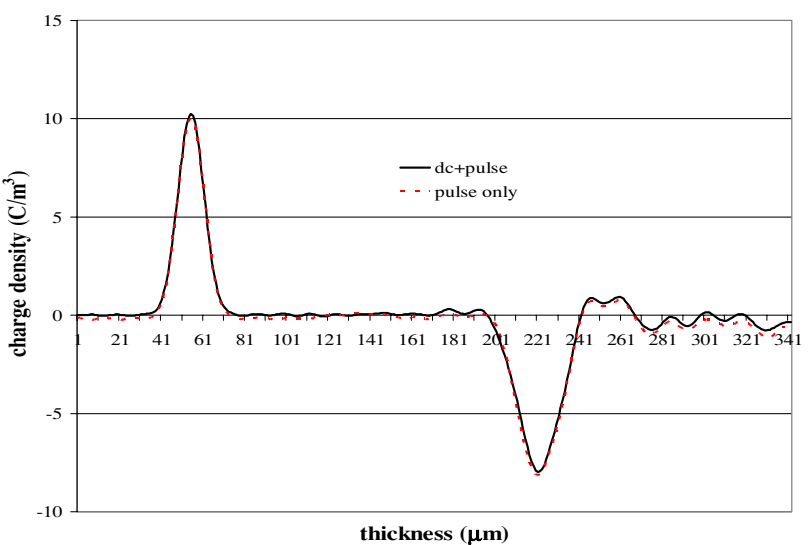

Figure 4. Comparison between calibration using pulse voltage only and that using de voltage.

is used during calibration, an equivalent dc voltage of $185 \mathrm{~V}$ should be added or subtracted from a normal calibration dc voltage depending on the polarity of the pulse voltage. It is worth stressing that both the applied voltage and pulse voltage make a contribution to the output signal. The contribution from the pulse voltage is often neglected. As a consequence, a significant error can be introduced. For example, based on figure 3, under the combination of an applied voltage of $10 \mathrm{kV}$ and a pulse voltage of $100 \mathrm{~V}$, the error introduced by neglecting the pulse voltage is only $0.35 \%$. However, under the combination of an applied voltage of $1 \mathrm{kV}$ and a pulse voltage of $600 \mathrm{~V}$, the error associated is $18.5 \%$; this error is quite high and cannot be ignored.

Figure 4 shows an example of the calibrated charge distribution in a $200 \mu \mathrm{m}$ thick low-density polyethylene (LDPE) sample using a $600 \mathrm{~V}$ pulse signal as calibration when a $2 \mathrm{kV}$ dc voltage is applied to the sample. For a comparison, a calibration based on both pulse and dc voltage is also shown. It is clear that both methods give almost the same distribution. The advantages of direct usage of the narrow pulse lie in its simplicity and little risk of producing bulk charge.

However, if the PEA system is not well constructed, the noise from the environment as well as the pulse generator can easily penetrate into the system. Consequently, the signal-tonoise ratio (SNR) of the response is poor. This problem can be overcome using a dc electric stress assuming that there is no bulk charge produced by the applied voltage. Since the magnitude of $X(f)$ is proportional to the amount of charge on the electrodes, $Y(f)$ can be increased to a level with a satisfactory signal-to-noise ratio. The second method is widely adopted because it does away with the requirement for the magnitude of the pulse generator to change over a wide range. However, the effect of the pulse voltage is always neglected. Good agreement can only be achieved in figure 4 when the pulse effect is taken into consideration.

\subsection{Sample with a trapped charge}

In some cases where charge is trapped either in the bulk or on the surfaces it is still possible to calibrate, so a quantitative charge distribution can be obtained. There are two methods available. The first solution to this problem is to use the same batch of samples without charge for calibration purposes. This 


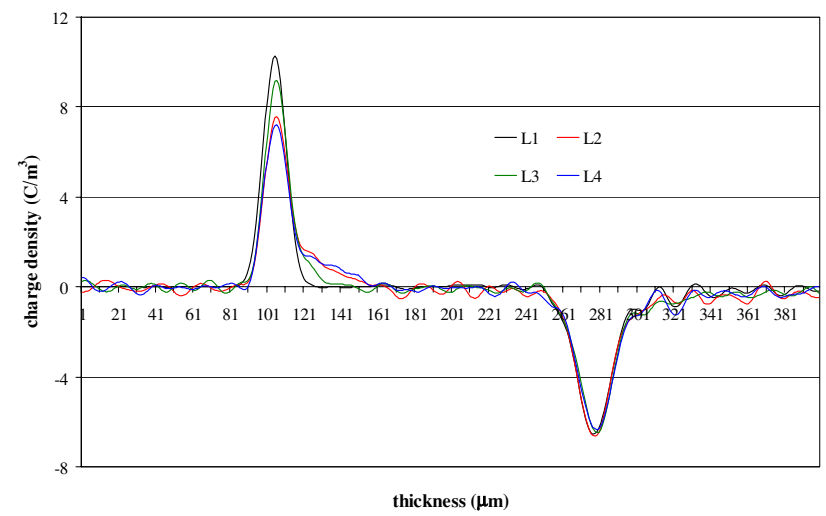

Figure 5. Effect of sample loading on charge distributions.

method has been used in the literature [24]. In theory, this method sounds very reasonable; however, in practice there are several interfaces involved in the PEA system which have a significant influence on the propagation of acoustic waves. Consequently, there are considerable variations in the output signal from sample to sample due to slight differences in sample assembling. It is, therefore, always desirable that the calibration and subsequent measurements are carried out on the sample without involving sample loading (placing a sample into a PEA system) and unloading (removing the sample from a PEA system). Figure 5 illustrates the results obtained from the same sample with different loadings under the same applied voltage (i.e. same pulse voltage and dc voltage) conditions. One can see that the magnitude of the output signal changes considerably. $L 1$ is a correctly calibrated charge distribution where its own signal has been used for calibration. However, when $L 1$ was used to calibrate the other three signals, charge distributions show significant differences. It appears that bulk charge is present in the sample, which we know is not true. One can easily understand now that a difference will be produced if either of them is used for calibration. $L 2, L 3$ and $L 4$ do not represent a correct charge distribution in the sample. Figure 6 demonstrates the influence of sample loading on charge and field distributions. Curve 1 in figures $6(a)$ and $(b)$ is a correct distribution for charge and electric field. In the case of using different loading signals as a calibration signal, it results in the 'presence' of either homocharge or heterocharge close to one of the electrodes, as shown by curves 2 and 3 . Again, curves 2 and 3 do not represent real charge and field distributions in the sample due to an inappropriate calibration. This problem can be readily discovered when a fresh sample is concerned. However, in the case of the sample in which there is a trapped charge, it is not an easy task to identify the problem.

The second method proposed below can be applied to the case where deeply trapped charge is involved. Charges can be roughly classified as fast and slow charges. 'Slow' charges are those which are essentially permanently trapped while 'fast' charges are those which escape from the traps very shortly after the dc voltage is removed. Charges trapped in the insulating material are often stable, i.e. slow charge. In a situation like this the calibration has to take two steps involving measurement with 'volts on' and 'volts off' (dc voltage). The 'volts on' measurement includes contributions from both fast and slow charges while the 'volts off' measurement

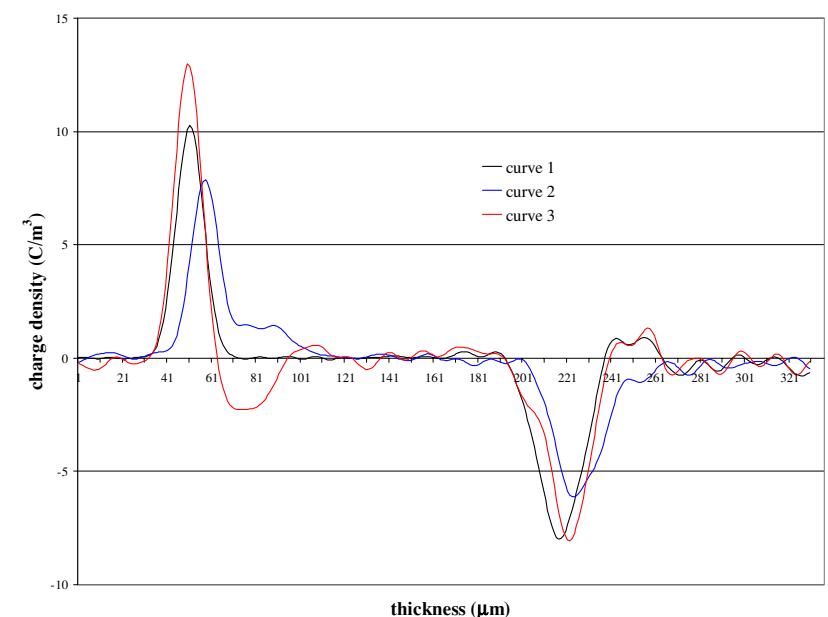

(a)

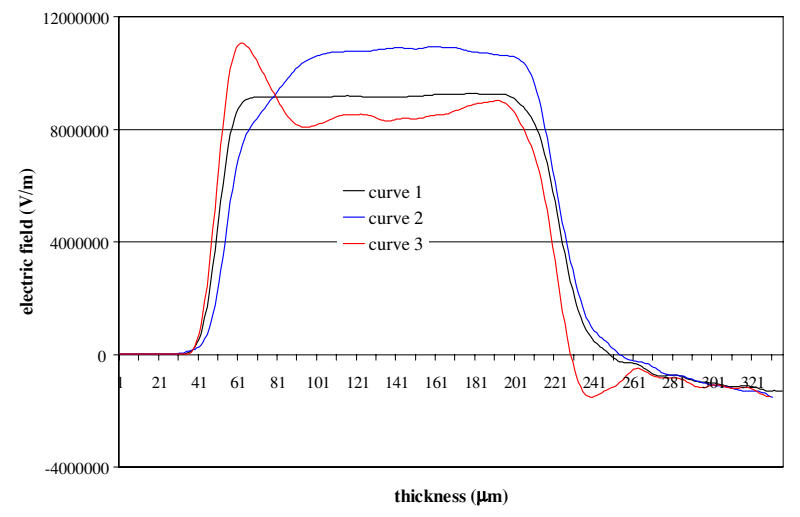

(b)

Figure 6. Influence of selecting different loadings as calibration signal on $(a)$ charge distribution and $(b)$ electric field distribution.

involves only slow charge. The subtraction between the two measurements gives the output signal which excludes the contribution from the trapped charge; in other words, it only represents the signal generated by the dc voltage; therefore, the calibration can be done in a similar way as described in section 3.1.

Assuming that the signals on the electrode near the transducer are $V_{1 \text {-on }}$ and $V_{1 \text {-off }}$, respectively corresponding to volts on and off, according to equation (2) we have

$$
\begin{gathered}
V_{1-\mathrm{on}}=K e_{\mathrm{p}}\left(\sigma+\delta+\sigma_{1}\right), \\
V_{1-\text { off }}=K e_{\mathrm{p}}(\sigma+\delta),
\end{gathered}
$$

where $\sigma$ represents the induced charge on the electrode due to the trapped charge in the sample, $\delta$ is the charge due to the pulse voltage and $\sigma_{1}$ is the capacitive charge due to the applied dc voltage.

By subtracting (7) from (6), the effect of the trapped space charge as well as the pulse voltage on the signals can be removed and this gives

$$
V_{1-\text { on }}-V_{1-\text { off }}=K e_{\mathrm{p}} \sigma_{1}
$$

Except for $K$, the rest of variables in the above equation can be either measured or are known from the applied voltage, therefore $K$ can be calculated. Once $K$ is known, the space charge in the bulk of the sample can be estimated based on equation (7). 


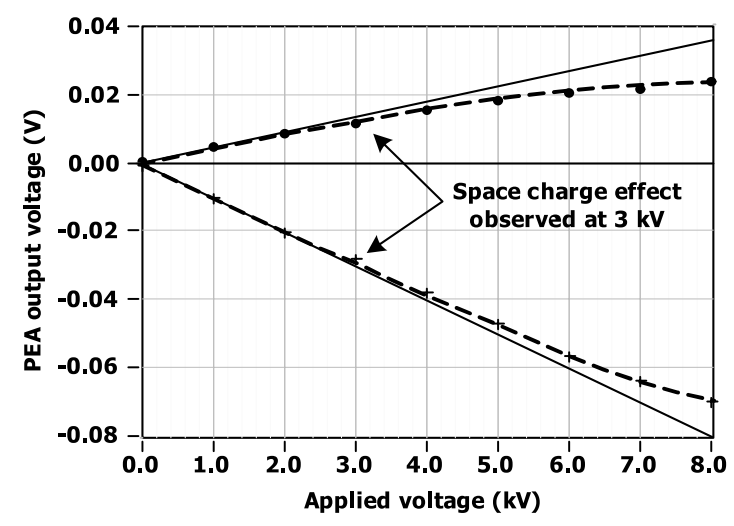

Figure 7. Result of step voltage test.

\section{Experimental results}

To validate the above calibration procedure, an experiment was devised. In this experiment an unaged LDPE of approximately $200 \mu$ m thick was used.

First, a step voltage test was conducted to determine the threshold voltage/stress at which the space charge effect is initiated in the sample of LDPE. Next, the sample is aged at $8 \mathrm{kV}$ for $60 \mathrm{~min}$ with the space charge being measured at regular intervals. This was done immediately after the step voltage test. After the ageing test, the voltage was removed and the space charge trapped in the sample was allowed to decay until it becomes reasonably stable; as before, the space charge was measured at regular intervals during this period of time. In the step voltage experiment, the voltage was raised from $0 \mathrm{kV}$ up to $8 \mathrm{kV}$ in step increments of $1 \mathrm{kV}$. The space charge measurement was taken at every step of the voltage increment.

Probably the easiest method to determine the presence of space charge in a material is to look at the interfacial stresses. Generally, the presence of homocharges or charges of the same polarity as the electrodes will result in the reduction of interfacial stress at the adjacent electrode. On the other hand, heterocharges or charges of opposite polarity to the electrodes will result in the enhancement of interfacial stress at the adjacent electrode.

As seen from figure 7 , the dotted lines which represent the interfacial stresses deviate negatively from the solid extrapolated trend lines which represent the interfacial stresses under no space charge condition. This suggests that homocharges initiate when the applied voltage is $3 \mathrm{kV}$. Hence a suitable signal of calibration is the signal measured at $2 \mathrm{kV}$. When an acoustic signal travels through a lossy material, its intensity attenuates. This results in different voltage magnitudes at the two electrodes, leading to different slopes with the applied voltage.

The result of the ageing experiment, as shown in figure 8, indicates homocharges build up in the vicinity of both electrodes. Note that the space charge is already observed at the beginning of the experiment. This further supports the result of the step voltage test. The origin of these homocharges is believed to be charge injected and subsequently trapped near the electrodes. It was observed that the amount of charge build-up increases with ageing duration.

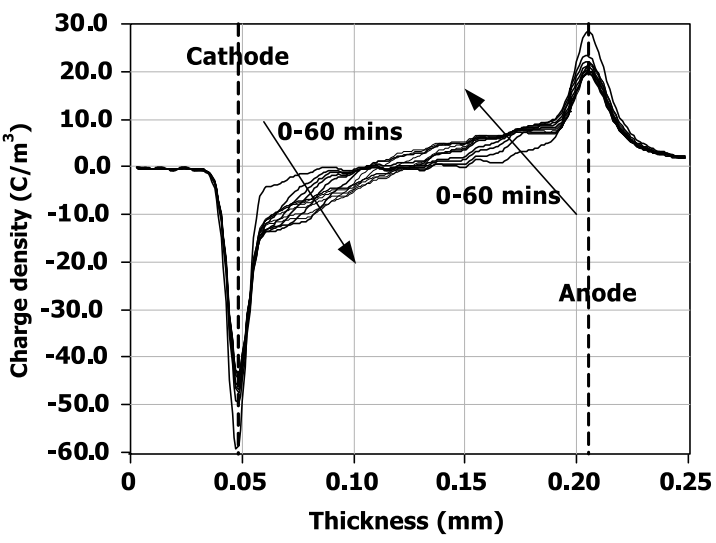

Figure 8. Result of ageing test showing the result of the decay test conducted over $15 \mathrm{~min}$.

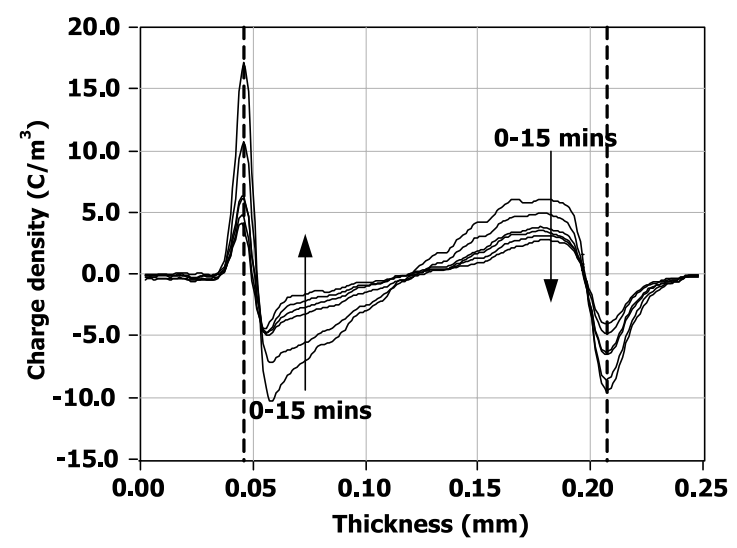

Figure 9. Result of decay test.

As our main purpose here is to produce a sample which has trapped charges inside, the ageing test was terminated at $60 \mathrm{~min}$. The charge decay in the aged sample was monitored as shown in figure 9. It can be seen that there is a significant reduction in the amount of homocharge trapped in the material initially. A steady reduction in the rate at which the space charge decays can be observed until it becomes reasonably stable after $15 \mathrm{~min}$.

Since the space charge profile after $15 \mathrm{~min}$ of charge decay is reasonably stable, it satisfies the condition that the charge is trapped reasonably deep and as such can be considered as a sample with trapped charge. Therefore, the method proposed in section 3.2 can be applied.

It should be stressed that in order to obtain suitable volts on signal, the applied voltage must be high enough so as to yield a signal of acceptable SNR. However, it is also important that the magnitude of the applied voltage should be low so that the charge originally accumulated is not significantly affected. Furthermore, applying too high a voltage may also be likely to cause fast charge accumulation in the sample. A suitable voltage for the present sample was found, based on the step voltage test, to be approximately $1 \mathrm{kV}$.

A comparison of calibration signals between the signal obtained during the initial step voltage test (solid line) and the signal obtained using the procedure described in section 3.2 (dotted line) is shown in figure 10. For the latter, 


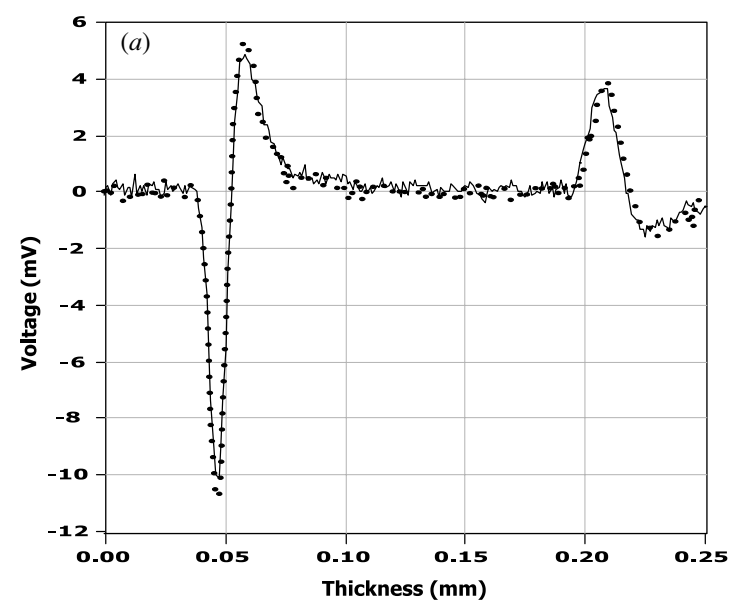

Figure 10. Comparison of calibration signals between the signal obtained during the initial step voltage (solid line) and that obtained in the presence of trapped charge (dotted line).

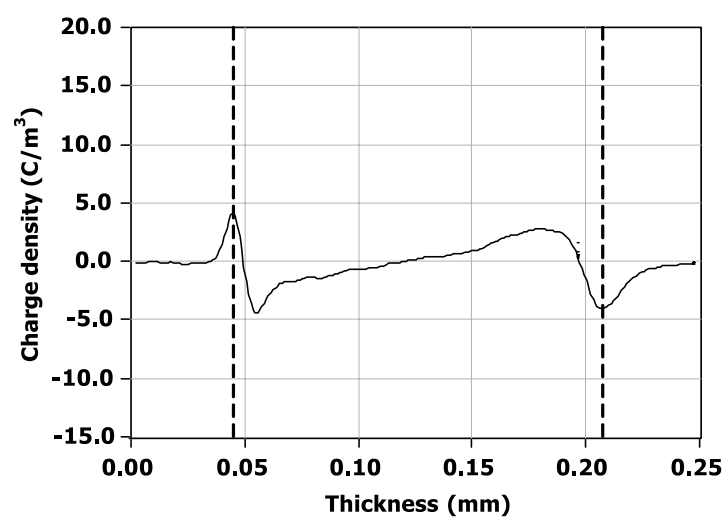

Figure 11. Trapped charge in the sample calculated using the proposed method.

a measurement was performed on the sample after it had decayed for $15 \mathrm{~min}$, then a $1 \mathrm{kV}$ dc voltage was applied to the sample.

It can be seen that there is little difference between the two signals, even to the extent that they almost overlap each other when put together. This implies that there is little fast charge accumulation in the sample when a $1 \mathrm{kV}$ dc voltage was applied. More importantly it validates the procedure described earlier.

Using the calibration signal obtained we have processed the data obtained from the decay test. Not surprisingly, the result is almost identical to that processed from the initial calibration signal as shown in figure 11.

\section{Discussion}

The PEA system discussed above only includes the effect of the sensor and the amplifier. In practice, averaging output signal over a couple of hundred times is often used to increase the signal-to-noise ratio. This is generally done through a digitizing scope or a data acquisition card. When data are processed a further filter may be employed to remove noise. Fortunately, all the systems used here are LTI systems. Therefore, the whole PEA system can be viewed as several LTI systems cascaded together and therefore is still an LTI system. The calibration procedures described above can be adopted.

Quantitative analysis of the space charge distribution is possible providing a full understanding of calibration carried out on the system. In a well-constructed PEA system, we have demonstrated that it is possible to use the pulse voltage to calibrate charge distribution in the sample where there is no charge initially. The pulse voltage alone provides an easy and straightforward calibration and runs little risk of producing any bulk charge that may affect the calibration. However, in majority of space charge measurements, a low dc voltage is applied in order to perform a calibration. In this case, we have demonstrated that the effect of the pulse voltage needs to be taken into consideration, depending on the ratio of the equivalent dc voltage of the pulse voltage and the applied dc voltage. The influence of the pulse will depend on several factors such as the magnitude of the pulse, pulse width and sample. Care must be taken when a low dc voltage is involved in calibration as both the pulse voltage and applied dc voltage produce capacitive charge on the electrodes. The influence of the pulse can be neglected only if the equivalent dc magnitude of the pulse voltage is 20 times less than the magnitude of the applied dc voltage for calibration (this leads to less than $5 \%$ error). An alternative procedure has been proposed by Hole et al [25] to eliminate the effect of the pulse. Two measurements by applying successively positive pulse and negative pulse with the same amplitude have to be carried out and subtracting the two measurements results in the removal of the second-order contribution from the pulse voltage. It has to be pointed out that the method we propose is much simpler and easy to implement in practice.

Calibration for a sample containing trapped charge has not been properly addressed before and a two-step procedure has been proposed here. Experimental evidence illustrates that the proposed method is viable; this is of particular interest in the application of electrets.

Although it is not considered for the sake of simplicity, we should point out that $K$ in the equations above may change with position depending on the material in consideration. When an acoustic pulse travels through a material it will interact with the material. The absorption and dissipation of the acoustic energy into heat or other forms of energy are the major reasons for attenuation. On the other hand the dispersion may be caused by the scattering of the acoustic waves (due to densely distributed inhomogeneities and frequency dependence of material constants such as elastic modulus). As a result, the acoustic pulse will decrease in magnitude and broaden in width. This effect cannot be neglected if a thick sample is in question. The spatial dependence of $K$ may result in different charge amplitudes seen on the two electrodes when they should be the same in the case of absence of space charge. A signal processing algorithm has been proposed [23] which recovers the signal.

\section{Conclusions}

Calibration is an important process in the quantitative analysis of the space charge in solid dielectrics. In the PEA technique, the pulse voltage effect on the quantitative charge distribution has often been largely ignored. This paper has demonstrated the importance of the pulse voltage involved in the charge 
calibration process. We have also proposed a method for calibration when the trapped charge is present in the sample. The following conclusions may be drawn.

In the case of a well-constructed PEA system, the pulse voltage alone can be used as a calibration signal; it avoids the worry of possible bulk charge produced by the applied dc voltage.

The effect of the pulse voltage needs to be considered where an accurate charge distribution is intended. A significant error can be introduced if low dc voltage and high pulse voltage are used for charge density calibration.

The proposed calibration method for the sample with trapped charge is very effective and the influence of the pulse voltage is cancelled out, leading to accurate charge distribution.

\section{References}

[1] Zhang Y, Lewiner J, Alquie C and Hampton N 1997 Evidence of strong correlation between space charge buildup and breakdown in cable insulation IEEE Trans. Dielectr. Electr. Insul. 4 778-83

[2] Liu Z, Liu R, Wang H and Liu W 1989 Space charge and initiation of electrical trees IEEE Trans. Electr. Insul. 24 83-9

[3] Li Y, Yasuda M and Takada T 1994 Pulsed electroacoustic method for measurement of charge accumulation in solid dielectrics IEEE Trans. Dielectr. Electr. Insul. 1 188-95

[4] Li Y 1994 Space charge measurement in lossy solid dielectric materials $P h D$ thesis Masashi Institute of Technology, Japan

[5] Lewiner J 1986 Evolution of experimental techniques for the study of the electrical properties of insulating materials IEEE Trans. Electr. Insul. 21 351-60

[6] Sessler G M, West J E and Gerhard G 1982 High resolution laser pulse method for measuring charge distributions in solid dielectrics Phys. Rev. Lett. 48 563-6

[7] Cherifi A, Abou-Dahka M and Toureille A 1992 The validation of the thermal step method IEEE Trans. Dielectr. Electr. Insul. 27 1152-8

[8] Lang S B and Das-Gupta D K 1986 A new technique for determination of spatial distribution of polarisation and space charge in polymer electrets J. Appl. Phys. 59 2151-60

[9] Mazzanti G, Montanari G C and Dissado L A 2005 Electrical ageing and life models: the role of space charge IEEE Trans. Dielectr. Electr. Insul. 12 876-90

[10] Fleming R J 2005 Space charge profile measurement techniques: recent advances and future directions IEEE Trans. Dielectr. Electr. Insul. 12 967-78
[11] Chen G 2005 Space charge in solid dielectrics ISH2OO5 (Beijing, China)

[12] Mizutani T 1994 Space charge measurement techniques and space charge in polyethylene IEEE Trans. Dielectr. Electr. Insul. 1 923-33

[13] Brown M A, Chen G, Davies A E, Dissado L A and Norman P A 2000 Space charge characterization in aged LDPE amalgamated insulation regions from underwater telecommunication systems IEEE Trans. Dielectr. Electr. Insul. 7 346-52

[14] Li Y, Takada T, Miyata H and Niwa T 1993 Observation of charge behaviour in multiply low-density polyethylene J. Appl. Phys. 74 2725-30

[15] Chen G, Tanaka Y, Takada T and Zhong L 2002 Effect of polyethylene interface on space charge formation IEEE Trans. Dielectr. Electr. Insul. 11 113-21

[16] Ho Y F F, Chen G, Davies A E, Hampton R N, Swingler S G and Sutton S J 2001 Do semicons affect space charge? ICSD'01 (Eindhoven, The Netherlands) pp 105-8

[17] Chen G, Tay T Y G, Davies A E, Tanaka Y and Takada T 2002 Electrodes and charge injection in low-density polyethylene - an experimental investigation using the pulsed electroacoustic technique IEEE Trans. Dielectr. Electr. Insul. 8 867-73

[18] Ho Y F F, Chen G, Davies A E, Swingler S G, Sutton S J and Hampton R N 2003 Effect of semiconducting screen on the space charge dynamic in XLPE and polyolefin insulation under dc and $50 \mathrm{~Hz}$ ac electric stresses conditions IEEE Trans. Dielectr. Electr. Insul. 10 393-403

[19] Chong Y L, Chen G, Hosier I L, Vaughan A S and Ho Y F F 2005 Heat treatment of cross-linked polyethylene and its effect on morphology and space charge evolution IEEE Trans. Dielectr. Electr. Insul. 12 1209-21

[20] Maeno T, Futami T, Kushibe H, Takada T and Cooke C M 1988 Measurement of spatial charge distribution in thick dielectrics using the pulsed electroacoustic method IEEE Trans. Electr. Insul. 23 433-9

[21] Zhu Y, Tu D and Takada T 2000 Mathematical analysis and interpretation of pulsed electro-acoustic system 6th ICPADM (Xian, China) pp 63-6

[22] Proakis J C and Manolakis D G 1996 Digital Signal Processing-Principles, Algorithms, and Applications 3rd edn (Englewood Cliffs, NJ: Prentice-Hall)

[23] Vazquez A, Chen G, Davies A E and Bosch R 1999 Space charge measurement using PEA technique and signal recovery J. Eur. Ceram. Soc. 19 1219-22

[24] Maeno T 1999 Calibration of the pulsed electroacoustic method for measuring space charge density Trans. IEE Japan 119-A 1114-19

[25] Hole S, Ditchi T and Lewiner J 2003 Non-destructive methods for space charge distribution measurements: what are the differences? IEEE Trans. Dielectr. Electr. Insul. 10 670-7 